\title{
Research on practice teaching of IOT application based on fresh agricultural product logistics
}

\author{
Yongjun Zhang ${ }^{1,2, ~ a, ~}{ }^{*}$, Shenggang $\mathrm{Wu}^{1,2}$ and Xugang $\mathrm{Zhu}^{1,2}$ \\ ${ }^{1}$ Shandong Institute of Commerce and Technology, Jinan 250103, China \\ ${ }^{2}$ Intelligent Information Engineering Technology Research and Development Center of Universities \\ in Shandong Province, Jinan 250103, China \\ aayj420177@163.com
}

\begin{abstract}
Practice teaching is a very important part of the whole teaching system. It plays a significant role in training and improving the practical ability and creative ability of college students. In view of the problems existing in applying teaching on the Internet of things, a set of modular practical teaching system of IOT application based on fresh agricultural products transportation has been proposed. The business processes covered by it with the technology support of the Internet of things, such as farm production, picking, packaging, transportation and sale. Through the study of the teaching system, the discrete knowledge can be systematized. After several years of teaching practice, the system is conducive to training students in the field of fresh agricultural logistics by applying the technology of the Internet of things.
\end{abstract}

Keywords: Internet of things, fresh agricultural products, logistics system, cold chain.

\section{Introduction}

Internet of things (IOT) application [1] is a new type of professional launched by the Ministry of education to serve the strategic emerging industries in China. Since 2010, there have been more than 700 colleges and universities in China, which have opened the Internet of things related courses [2]. After seven years of teaching practice, networking engineering training mode has made great progress in the curriculum and talent, but at this stage of graduates who are major in IOT engineering cannot meet the development needs of the IOT industry, most of the graduates can only support the primary work outside the industry, and to the innovative talents of engineering application for the Internet of things planning and design, development and management is a shortage. The university is still in the initial stage of the Internet of things education, and the personnel training system is not perfect enough, especially the practice teaching link is still relatively weak. In view of the existence of things engineering problems in the teaching of architecture, to construct an IOT technology teaching system based on fresh agricultural logistics will bring more specific technologies for students to learn and practice. Therefore, students who armed with such knowledge can satisfy industry IOT development of fresh cold chain logistic.

\section{The basis of division of teaching system in IOT application}

The teaching module is a combination of teaching activities carried out around specific topics and specific skills training [3, 4]. Modular according to the professional knowledge structure and personnel training objectives, the content closely linked, internal logic, the same category of skills in the curriculum as a module to carry out teaching [5]. For the students of the Internet of things engineering, it is of great practical significance to practice the modularization of the teaching system. 
The whole teaching system and concerned knowledge modules are throughout the all fresh agricultural supply chain and involve many regulations in that field, which is demonstrated in figure 1 . In every stage of cold chain, IOT technologies will solve specific problems and improve efficiency of concerned operations.

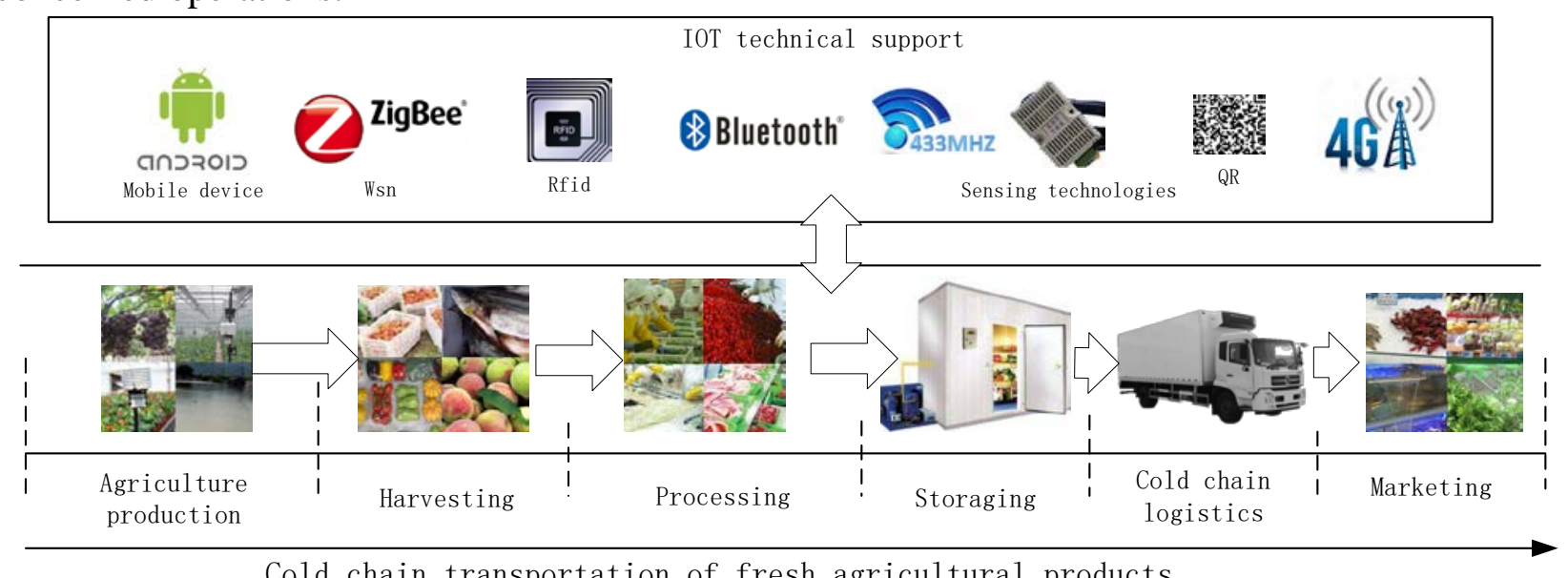

Cold chain transportation of fresh agricultural products

Fig. 1 Cold chain of fresh agriculture products and its concerned IOT technical support

Practice teaching system is a systematic project, must follow certain principles to develop the scientific and operational teaching system. When partitioning a module, it should in accordance with the following four principles.

(1) Practical principle. The practical teaching system should be practical, specific and efficient. In the module, to analyze the characteristics and experiment each experimental course should meet the objectives and requirements, focusing on the same module curriculum knowledge integration and the relationship between before and after strengthening the students' comprehensive ability to use knowledge, practical ability and innovation ability.

(2) Systematic principle. The systematic studies the knowledge architecture of the Internet of things, and divides the modules from the perspective of the Internet of things.

(3) Follow the law of teaching. Practice teaching should conform to the law of education and teaching, step by step, from the cognitive practice teaching to the professional skills practice teaching, and then to the comprehensive and innovative practice teaching, gradually improve the students' practical ability.

(4) In line with the actual situation of the college. The practical teaching system should be worked out according to the school teachers' strength and the school laboratory conditions.

\section{The division of practical teaching module}

When dividing a module, it is necessary to study the architecture of the Internet of things and make clear the main technologies and relationships among the layers. IOT refers to the sensors, radio frequency identification information sensing equipment, according to the agreed protocol to connect any object to the Internet for information exchange and communication, to achieve a network intelligent identification, monitoring and management. Its main characteristic is to obtain the material world through sensors, RFID and other forms of information, for transfer and the interaction of information through the Internet, mobile communication network, using intelligent computing technology for information analysis and processing, improve the perception of the physical world, the realization of intelligent decision-making and control [6]. The architecture of the Internet of things [7, 8] can be divided into information layer, network layer, data processing layer and application layer according to the information generation, transmission, processing and application. This paper will partition the teaching module into five sections, and the specific modules are as follows.

\subsection{Cognitive practice teaching module}

The module of cognitive practice teaching mainly includes the introduction of computer and the introduction of the Internet of things, the practical teaching of these two courses. The module is 
composed of teaching objective and related technology to enable students to master the computer hardware, software, computer networks, Internet of things, clear the meaning and the relationship between professional courses, which is a solid foundation for the follow-up study. The practice teaching mainly combines teacher demonstration with the student's operation, and makes the abstract theory become vivid by experiment demonstration, and let students do hands-on work to arouse students' thirst for knowledge. Computer technology is the basis of networking technology, teaching computer introduction, should increase the knowledge of things, not only to improve the introduction of computer knowledge, but also a solid foundation for students to learn IOT. Through the study of these two courses, let students understand the connection and the difference between computer science and technical specialty and Internet of things engineering, which is beneficial to students' academic planning and career planning.

\subsection{Program design and algorithm analysis practice teaching module}

Program design and algorithm analysis practice teaching module mainly includes Java language programming and data structure of the two courses of practice teaching. The practical teaching of the module aims to enable students to master the Java language, data structure and algorithm design knowledge, focusing on improving programming ability and algorithm design capabilities. Java language program design practice teaching of the basic knowledge of grammar, the gradual introduction of classic and interesting and close contact with the daily life of the case, to guide the students from the point of view of problem solving, learning programming knowledge, and cultivate students' interest in programming and programming thinking, improve programming ability of students. Data structure allows students to master the logical structure, physical structure and related algorithms of the classical data structure. The emphasis is on the organizational structure and algorithmic implementation of the data.

\subsection{Perception layer practice teaching module}

The sense layer is mainly responsible for information collection and short distance transmission, which is the core layer of the Internet of things system. The perception layer courses of our college have electronic circuits, digital logic, computer composition principle, microcomputer principle and interface, embedded system, signal system, sensor technology, wireless sensor network, data acquisition technology, radio frequency identification technology, ZigBee technology and other courses. This module has many practical teaching courses, a large time span, and a close link between courses. Therefore, we must pay attention to the links between courses in the content of experiment teaching. According to the relation among courses, the module practice teaching can be subdivided into hardware courses group and data acquisition course group.

\subsection{Network layer practice teaching module}

The network layer is mainly responsible for the transmission of information. The content of the module enables students to clearly understand the process of transmission of information, principles and applications, so that students can choose appropriate communication methods according to actual needs, and build the system of the Internet of things. The courses offered for the network layer include communication principle, computer network principle, TCP/IP protocol analysis, networking technology and network information security technology. Through the Internet network technology curriculum learning, to enable students to build communication network is feasible, and can make students get knowledge through learning, and apply it in practice.

\subsection{Data processing layer practice teaching module}

The data processing layer stores, processes and makes intelligent decision service for the massive data transferred from the network layer. Through this module student's basic process, working principle and main methods of data processing control networking project, and use this knowledge to propose a specific data processing scheme for typical networking applications. Finally, the law application will find valuable in practice and have practical application value. 


\section{Implementation of modular practice teaching system}

In order to achieve modular teaching practice, teaching processing must follow the laws of teaching, formulate an implementation plan step by step, from the shallow to the deeper, the whole process of practice teaching in general practice teaching according to the cognitive order module, algorithm and program design of the practice teaching module, teaching module, perception layer, network layer, application layer module and data processing layer practice teaching module development. As each module contains multiple courses, the time between each module is partially overlapped. In order to further improve the students' practical ability and comprehensive ability to apply knowledge.

\section{Conclusions}

The subject of Internet of things is an interdisciplinary subject which includes computer major, communication specialty and automation specialty. It is a typical subject based on engineering application. Therefore, practice teaching plays a vital role in the quality of personnel training. This article aims at the present the problems existing in the teaching of Internet of things engineering, and a set of modular teaching system based on the practice of IOT application for fresh agricultural products is being put forward.

\section{Acknowledgments}

The research was supported by intelligent information engineering technology research and development Center of colleges and universities in Shandong Province, and it was also sponsored by project "The intelligent monitoring equipment of fish water-free dormancy keep live transportation (J15LN52)" and project "Research and practice of "platform sharing and module distributary" talents training mode in computer specialty group under the background of credit system" form Shandong Provincial Education Department.

\section{References}

[1] Z Lan, X Zhao, J Liu. A Yuan. Hanraads, R.A. Lupton, Discussion on communication engineering specialty professional oriented the internet of things, J. China Modern Educational Equipment, 2013(4)102-109.

[2] Duxue-Feng. Discussion on the specialty construction in the Internet of Things of Higher Vocational Education, Journal of Sanmenxia Polytechnic, 2012(5)68-73

[3] Z Zhang , C Cheng. Design of Polytechnic College Practice Training Base Management System Based on Internet of Things, Applied Mechanics \& Materials, 2014, 496-500:2224-2227.

[4] J Mei, X Yang, Y Tang. Practice of talent training by cooperating of production, teaching, research and use in specialty group of internet of things at higher vocational colleges: The case of Wuxi City College of Vocational Technology, 2015(2):27-34.

[5] H Yan, H Hu. Research and realization on CDIO teaching experimental system based on RFID technique of Internet of Things, International Conference on Mechatronic Science, 2011:841-844.

[6] D Pereţianu, C Giurcăneanu, A Deleanu. Intelligent Decision-Making Service Framework Based on QoS Model in the Internet of Things, International Symposium on Distributed Computing \& Applications to Business, 2012 , 22 (2) :103-107.

[7] A Hakansson, R Hartung, An Infrastructure for Individualized and Intelligent Decision-making and Negotiation in Cyber-physical Systems, Procedia Computer Science, 2014 , 35 :822-831.

[8] LY Chu, XQ Chen, S Dang. Design and Implementation of Supply Chain Integrated System Based on Internet of Things, Applied Mechanics \& Materials, 2013, 433-435:2395-2400. 